\title{
A High Convection Dominated Thermally Driven Problems
}

\author{
Blanca Bermudez-Juarez, Jose David Alanís*, Alejandro Rangel-Huerta, W. Fermín Guerrero \\ Division of Technologies of Information, Technological University of Puebla, Puebla, México.
}

How to cite this paper: Blanca Bermudez-Juarez, Alejandro Rangel-Huerta, Jose David Alanís, W. Fermín Guerrero. (2020) A High Convection Dominated Thermally Driven Problems. Journal of Applied Mathematics and Computation, 4(4), 254-260. DOI: 10.26855/jamc.2020.12.017

Received: November 25, 2020 Accepted: December 18, 2020

Published: December 28, 2020

${ }^{*}$ Corresponding author: Jose David Alanís, Division of Technologies of Information, Technological University of Puebla, Puebla, México.

Email: david.alanis@utpuebla.edu.mx

\begin{abstract}
In this paper, we are performing a numerical simulation of unsteady plumes for driven thermal high convection dominated flows. These problem results of practical interest, in order to determine the efficiency of heat storage, flow conditions inside electronic components, determining the durability of the equipment, etc. Results for Rayleigh numbers in the range of $10^{6} \leq \mathrm{Ra} \leq 10^{11}$ are presented. In this case, an intermittent spatial transition from laminar to turbulent flows takes place; therefore, high accuracy of spatial and time discretization is required. In this paper, we use finite differences and a fixed-point iterative method. This method has shown to be robust enough to handle high Rayleigh numbers been able to simulate these kinds of phenomena. When working with high Rayleigh numbers and since we have to arrive to a large value of $t$ (time), at each iteration in time, we have to solve a very large system of linear equations.
\end{abstract}

\section{Keywords}

Buoyant Plumes, Boussinesq Approximation, Rayleigh Number, High Convection Dominated Flows

\section{Introduction}

We present here a numerical simulation of unsteady plumes for driven thermal high convection dominated flows. The Boussinesq system in the stream function-vorticity formulation is used, in our case, to model this kind of flows. Finite Differences are used, and then, the linear systems of equations resulting after time and space discretization are solved via a fixed-point iterative process previously reported in the bibliography. The numerical method used here has been previously reported in Nicolás and Bermúdez [1] for mixed convection and in Báez and Nicolás [2] for natural convection in tilted cavities. This scheme has shown to be robust enough to handle high Rayleigh numbers and different aspect ratios (ratio of the height to the width) of the cavity. The evolution of the flows we are dealing with in this paper depends mainly on the variation of the parameters. The buoyancy forcing of the flow can be characterized by the Rayleigh number $\mathrm{Ra}$, which in the case of this work, is taken in the range $10^{6} \leq R a \leq 10^{11}$. For this range of the Rayleigh numbers, numerical results are not easy to obtain, since in this case we have a highly convection-dominated thermally driven problem.

An extensive study of buoyant plumes over a line heat source in the interior of a rectangular cavity is presented by Desrayaud and Lauriat [3]. In Minev et al. [4], a splitting procedure combined with spectral element discretization is developed and preliminary results of the direct numerical simulation of unsteady plumes in a square cavity induced by a finite-area heat source on its bottom are performed.

Large-eddy simulations are performed in Basiaans et al. [5]. They found that the flow deviates from laminar at $\mathrm{Ra}=10^{8}$. They worked for Rayleigh numbers in the range of $10^{8} \leq R a \leq 10^{9}$. The 3D case is also considered here. In Bill Jr. and Gebhart [6], transition to turbulence is examined in their experiments. Turbulent plane plumes are reported by an expe- 
rimental study in Rouse et al. [7].

In Manar et al. [10], the authors say latent heat storage represents a promising technique to achieve net zero energy buildings. They investigate the behavior of phase change material (PCM) inside a rectangular enclosure, which represents the geometry of a latent heat storage system. In Ahmed et al. [11], authors proposed a non-intrusive reduced order modeling framework that is best suited for unsteady flows, where the convective mechanisms are more predominant than the diffusive ones. The approach is based on principal interval decomposition to parse the data over time to learn more localized dominant structures. In Prasopchingchana [12], effects of cavity aspect ratios and cavity inclination angles to natural convection in a rectangular cavity are numerically investigated. Investigation is performed at the Rayleigh number equal to $10^{4}$, the cavity aspect ratios from 1 to 50 and the cavity inclination angles from 0 to $180^{\circ}$. In Mamourian et al. [13], authors analyzed mixed convection heat transfer inside lid-driven cavities with a wavy surface. Mastiani et al. [14] and $\mathrm{Yu}$ et al. [15] conducted numerical simulations of mixed convection of nanofluids in lid-driven square enclosures. Lei et al. [16] presents a comprehensive analysis of effects of porous fins on mixed convection heat transfer in lid-driven square cavities, where the top lid has the two-way movement

\section{Methods}

\subsection{Mathematical Model}

Let $\Omega \subset R^{n}(N=2,3)$ be the region of a non-steady, viscous, incompressible flow, and $\Gamma$ its boundary. Under the hypothesis of the Boussinesq approximation, this kind of fluids may be modeled by the following adimensional system:

$$
\begin{gathered}
\mathrm{u}_{\mathrm{t}}-\nabla^{2} \mathbf{u}+\nabla p+(\mathbf{u} \cdot \nabla) \mathbf{u}=\frac{R a}{P r} \theta \boldsymbol{e} \\
\nabla \cdot \mathbf{u}=0 \quad(b) \\
\theta_{t}-\frac{1}{P r} \nabla^{2} \theta+\mathbf{u} \nabla \theta=0 \quad(c)
\end{gathered}
$$

The parameters $R a$ and $P r$ are the Rayleigh and the Prandtl number respectively, which are given by: $R a=$ $\frac{\beta l^{3} \kappa g p_{0}^{2}}{\mu^{3} c_{p}}\left(T_{1}-T_{0}\right), \operatorname{Pr}=\frac{\kappa}{\mu c_{p}}$ here $T_{0}$ and $T_{1}$ are reference temperatures, $T_{0}<T_{1}$, which may be the temperatures of the side walls when the region is a rectangular cavity. The adimensional temperature $\theta$ is given by: $\theta=\frac{T-T_{0}}{T_{1}-T_{0}}$.

This system must be provided with appropriate initial and boundary conditions:

$$
\mathbf{u}(\mathbf{x}, 0)=\mathbf{u}_{\mathbf{0}}(\mathbf{x}) \text { and } \theta(\mathbf{x}, 0)=\theta_{0}(\mathbf{x}) \text { in } \Omega ; \mathbf{u}=\mathbf{f} \text { and } \mathrm{B} \theta=0 \text { in } \Gamma, \mathrm{t} \geq 0
$$

where B is a boundary operator for the temperature and may involve Dirichlet, Neumann and mixed boundary conditions.

Restricting ourselves to a bi dimensional region $\Omega$, taking the curl in both sides of equation (1a) and keeping in mind that

$$
u_{1}=\frac{\partial \psi}{\partial y} \quad, \quad u_{2}=-\frac{\partial \psi}{\partial x},
$$

which follows from (1b), with $\psi$ the stream function and $\left(u_{1}, u_{2}\right)=\mathbf{u}$; the component in the $\mathbf{k}=(0,0,1)$ direction, we get the following scalar system:

$$
\begin{array}{r}
\nabla^{2} \psi=-\omega \quad(a) \\
\omega_{t}-\nabla^{2} \omega+\mathbf{u} \cdot \nabla \omega=\frac{R a}{P r} \frac{\partial \theta}{\partial x} \\
\theta_{t}-\gamma \nabla^{2} \theta+\mathbf{u} \nabla \theta=0
\end{array}
$$

where $\gamma=1 / P r$ and $\omega$ is the vorticity, which, from $\omega \boldsymbol{k}=\nabla \times \mathbf{u}=-\nabla^{2} \psi \boldsymbol{k} \omega$ gives (3a) and $\omega=\frac{\partial u_{2}}{\partial x}-\frac{\partial u_{1}}{\partial y}$ also.

Then, system (3) becomes the Boussinesq approximation in stream function vorticity variables. The incompressibility condition (1b), by (2), is automatically satisfied and the pressure p has been eliminated. A translation of the boundary conditions in terms of the velocity primitive variable $\mathbf{u}$ to the $\psi-\omega$ variables has to be performed.

Following [8], $\psi$ is a constant function on solid and fixed walls; at the moving wall $y=b$, a constant function for $\psi$ is also obtained, then $\psi=0$ is chosen in $\Gamma$. By Taylor expansion of (3a) on the boundary, with $h_{x}$ and $h_{y}$ the space steps, one obtains:

$$
\omega(0, y, t)=-\frac{1}{2 h_{x}^{2}}\left[8 \psi\left(h_{x}, y, t\right)-\psi\left(2 h_{x}, y, t\right)\right]+O\left(h_{x}^{2}\right)
$$




$$
\begin{gathered}
\left.\omega(a, y, t)=-\frac{1}{2 h_{x}^{2}}\left[8 \psi\left(a-h_{x}, y, t\right)-\psi\left(a-2 h_{x}, y, t\right)\right]+O\left(h_{x}^{2}\right)\right) \\
\omega(x, 0, t)=-\frac{1}{2 h_{y}^{2}}\left[8 \psi\left(x, h_{y}, t\right)-\psi\left(a-2 h_{x}, t\right)\right]+O\left(h_{y}^{2}\right) \\
\omega(x, b, t)=-\frac{1}{2 h_{y}^{2}}\left[8 \psi\left(x, b-h_{y}, t\right)-\psi\left(x, b-2 h_{y}, t\right)\right]+O\left(h_{y}^{2}\right)
\end{gathered}
$$

where $h_{x}$ and $h_{y}$ denote the size of the spatial discretization in the $\mathrm{x}$ and $\mathrm{y}$ directions. It should be observed that the boundary values for $\omega$ are given by values in $\Omega$ and $\mathrm{t}>0$, still unknown, of the stream function $\psi$. This problem will be solved using a fixed-point iterative process.

\subsection{Description of Numerical Method}

The time derivatives with respect to $\omega$ and $\theta$ in (3) are approximated by the following second order approximation:

$$
f_{t}(x,(n+1) \Delta t) \approx \frac{3 f^{n+1}-4 f^{n}+f^{n-1}}{2 \Delta t}
$$

where $n \geq 1, x \in \Omega, \Delta t>0$ is the time step, and $f^{r} \approx f(\mathbf{x}, r \Delta t)$; at each time level $t=(n+1) \Delta t$, the following system in $\Omega$ is obtained:

$$
\begin{gathered}
\nabla^{2} \psi^{n+1}=-\omega^{n+1},\left.\quad \psi^{n+1}\right|_{\Gamma}=0 \\
\alpha \omega^{n+1}-\nabla^{2} \omega^{n+1}+\mathbf{u}^{n+1} \cdot \nabla \omega^{n+1}=\frac{R a}{P r} \frac{\partial \theta^{n+1}}{\partial x}+f_{\omega},\left.\omega^{n+1}\right|_{\Gamma}=0 \\
\alpha \theta^{n+1}-\gamma \nabla^{2} \theta^{n+1}+\mathbf{u}^{n+1} \nabla \theta^{n+1}=\left.f_{\theta} \quad B \theta^{n+1}\right|_{\Gamma}=0
\end{gathered}
$$

where $\alpha=\frac{3}{2 \Delta t}, f_{\omega}=\frac{4 \omega^{n}-\omega^{n+1}}{2 \Delta t}$, and $f_{\theta}=\frac{4 \theta^{n}-\theta^{n-1}}{2 \Delta t} ; \omega_{b c}$ denotes the boundary condition of $\omega$, B denotes the boundary operator for $\theta$ mentioned above, and $u_{1}$ and $u_{2}$ the components of $\mathbf{u}$ in terms of $\psi$ are given by (2).

Denoting $\left(\psi^{n+1}, \omega^{n+1}, \theta^{n+1}\right)$ by $(\psi, \omega, \theta)$ the following non-linear systems is obtained:

$$
\begin{gathered}
\nabla^{2} \psi=-\omega,\left.\quad \psi\right|_{\Gamma}=0 \\
\alpha \omega-\nabla^{2} \omega+\mathbf{u} \cdot \nabla \omega=\frac{R a}{P r} \frac{\partial \theta^{n+1}}{\partial x}+f_{\omega},\left.\quad \omega\right|_{\Gamma}=0 \\
\alpha \theta-\gamma \nabla^{2} \theta+\mathbf{u} \nabla \theta=\left.f_{\theta} \quad B \theta\right|_{\Gamma}=0
\end{gathered}
$$

To obtain $\left(\omega^{1}, \theta^{1}, \psi^{1}\right)$ in (6), a first order approximation for the time derivatives may be applied through a subsequence with a smaller time step; a stationary system is then also obtained.

Denoting by:

$$
\begin{gathered}
R_{\omega}(\omega, \psi) \equiv \alpha \omega-\nabla^{2} \omega+\mathbf{u} \nabla \omega-\frac{R a}{\operatorname{Pr}} \frac{\partial \theta}{\partial x}-f_{\omega} \\
R_{\theta}(\theta, \psi)=\alpha \theta-\gamma \nabla^{2} \theta+\mathbf{u} \nabla \theta-f_{\omega}
\end{gathered}
$$

Then, system (7) is equivalent, in $\Omega$, to:

$$
\begin{array}{rl}
\nabla^{2} \psi=-\omega, & \left.\psi\right|_{\Gamma}=0 \\
R_{\omega}(\omega, \psi)=0 & \left.\omega\right|_{\Gamma}=\omega_{b c} \\
R_{\theta}(\theta, \psi)=0 & \left.B \theta\right|_{\Gamma}=0
\end{array}
$$

To solve this system at each time level $(n+1) \Delta t$, the following fixed- point iterative process [9] is applied in $\Omega$ With $\left\{\theta^{0}, \omega^{0}\right\}=\left\{\theta^{n}, \omega^{n}\right\}$ given, solve "until convergence" on $\theta$ and:

$$
\begin{gathered}
\nabla^{2} \psi^{m+1}=-\omega^{m}, \quad \psi^{m+1}=0 \text { on } \Gamma \\
\theta^{m}-\rho_{\theta}\left(\alpha I-\gamma \nabla^{2}\right)^{-1} R_{\theta}\left(\theta^{m}, \psi^{m+1}\right) \\
B \theta^{m+1}=0 \\
\omega^{m+1}=\omega^{m}-\rho_{\omega}\left(\alpha I-\nabla^{2}\right)^{-1} R_{\omega}\left(\omega^{m}, \psi^{m+1}\right) \\
\omega^{m+1}=\omega_{b c}^{m+1} \text { on } \Gamma, \quad \rho_{\omega}>0
\end{gathered}
$$

and then take $\left(\omega^{n+1}, \psi^{n+1}, \theta^{n+1}\right)=\left(\omega^{m+1}, \psi^{m+1}, \theta^{m+1}\right)$.

With "until convergence" we mean until two consecutive values of $\theta$ and $\omega$, that is, $\theta^{m+1}$ and $\omega^{m+1}$ and $\omega^{m}$, differ 
by less than a certain tolerance, tol, given.

Then, at each iteration, three linear elliptic problems associated with the operators $\nabla^{2}, \alpha I-\gamma$, and , $\alpha I-\nabla^{2}$ have to be solved in $\Omega$. For the discretization of such problems, either finite elements or finite differences may be used, as long as rectangular domains, as in the case of this work, are considered.

\section{Results}

Our numerical experiments take place in a rectangular cavity with adiabatic side walls and isothermic base and top. For the temperature, the source in the bottom, collocated in the center, is taken as a smooth Gaussian hill: $\partial \mathrm{T} / \partial \mathrm{n}=\mathrm{e}^{\wedge}\left(-\mathrm{F}\left(\mathrm{x}-\mathrm{x} \_0\right)^{\wedge} 2\right)$ (with $\mathrm{x} \_0$ the center). On the top wall, zero temperature is prescribed. The heat source generates a convective plume that develops a free flotation flow that is deformed by confinement within the cavity. Under these conditions, turbulent flows and asymmetric patterns of temperature are generated as the Rayleigh number; an adimensional parameter that characterizes confined flows, increases. The cavity is supposed to be filled with air, so the Prandtl number considered here is $\operatorname{Pr}=0.71$.

In the figures shown, transition from laminar to turbulent flows, as well as changes in the lines of temperature patterns, for different Rayleigh numbers, may be observed. The adjustment or refinement of other parameters provides better resolution in the simulation, so different changes of phase in the composition of the flow vortices, can be observed.

In Figure 1, we show results for $\mathrm{Ra}=\llbracket 10 \rrbracket \wedge 6$, at $\mathrm{t}=0.05$ with $\mathrm{h}_{\mathrm{x}}=\mathrm{h}_{\mathrm{y}}=1 / 256$ and $\mathrm{t}=0.00001$. In the graph of the streamline function two vortices in counter flow, but of the same intensity, can be observed. The isotherms, in this case, are symmetric with respect to the source of heat, occupying only part of the cavity. This effect ends with symmetry breaking and transition to turbulent flow.
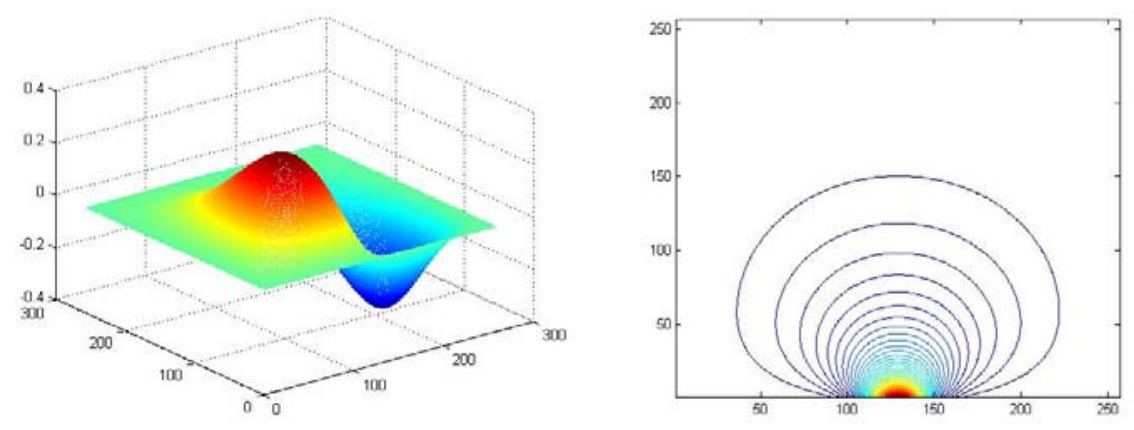

Figure 1. Stream function and isotherms for $R a=10^{6}, t=0.05, d t=0.00001, h_{x}=h_{y}=1 / 256$.

As the Rayleigh number increases, a turbulent flow structure is generated due to convective recirculation inside the cavity. In Figure 2, we show results for $R a=10^{7}$ at $t=0.05$ with $h_{x}=h_{y}=1 / 256$ and $\mathrm{t}=0.00001$. In this case, we can observe just one vortex in the graph of the streamline function with its maximum located at the center of the cavity. Nevertheless, the isotherms begin to show some asymmetry, bending towards the right wall, and presenting a fold at the left top part without filling the cavity. This indicates that the flow of heat is being transported by the movement of the fluid flow.

For the model of the cavity considered here, it is known that for Rayleigh numbers less than $2.8 \times 10^{7}$, the system is stable. In fact, the system presents a punctual attractor in the phase space.
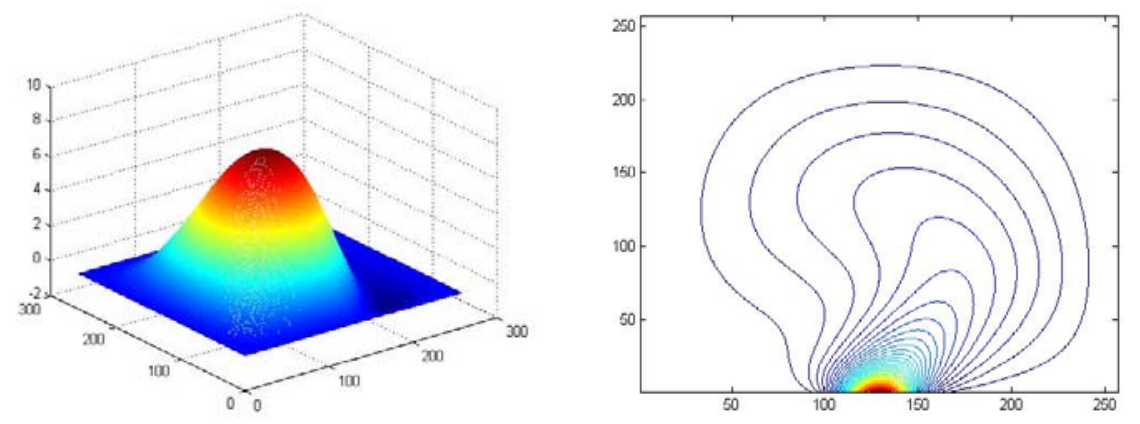

Figure 2. Stream function and isotherms for $R a=10^{7}, t=0.05, d t=0.00001, h_{x}=h_{y}=1 / 256$. 
We show, in Figure 3, results for $R a=10^{8}$ at $t=0.05$ with $h_{x}=h_{y}=1 / 256$ and $t=0.00001$. We can observe here the restoration of two vortices of counter flow, although one dominates the circulation and the other one is smaller. The isotherms present well-defined asymmetric contours with a plume to the right near the heat source and irregular contours in the top right of the cavity.
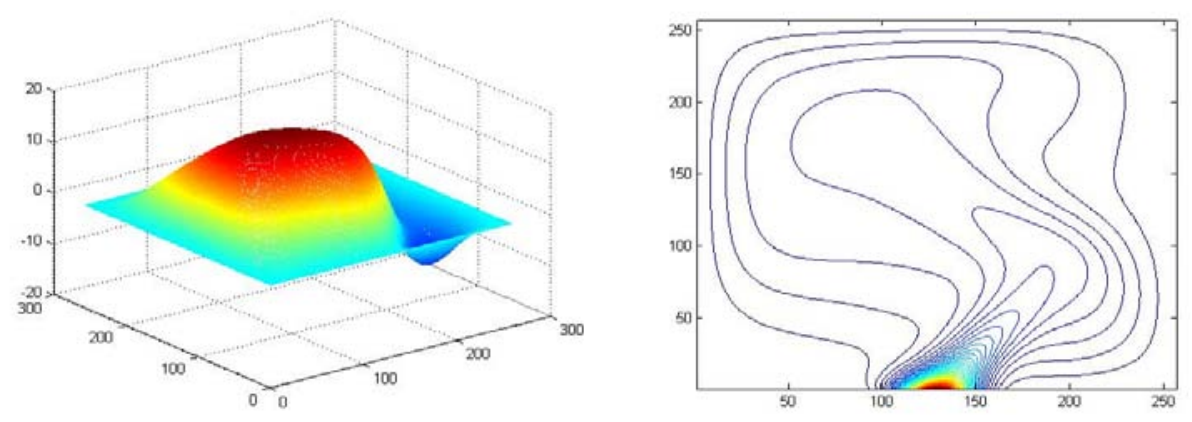

Figure 3. Stream function and isotherms for $R a=10^{8}, t=0.05, d t=0.00001, h_{x}=h_{y}=1 / 256$.

In Figure 4, we show results for $R a=10^{10}$ at $t=0.05$ with $h_{x}=h_{y}=1 / 256$ and $t=0.00001$. In this case, the dominant vortex loses its typical symmetry, modifying its amplitude. The temperature contours gain structure close to the source, with well-defined loops in the upper-right corner of the plume, separated from the structure of isotherms of the full cavity that prevails for Ra less than $10^{9}$. In this case, the pattern of the isotherms presents two local regions of bifurcation
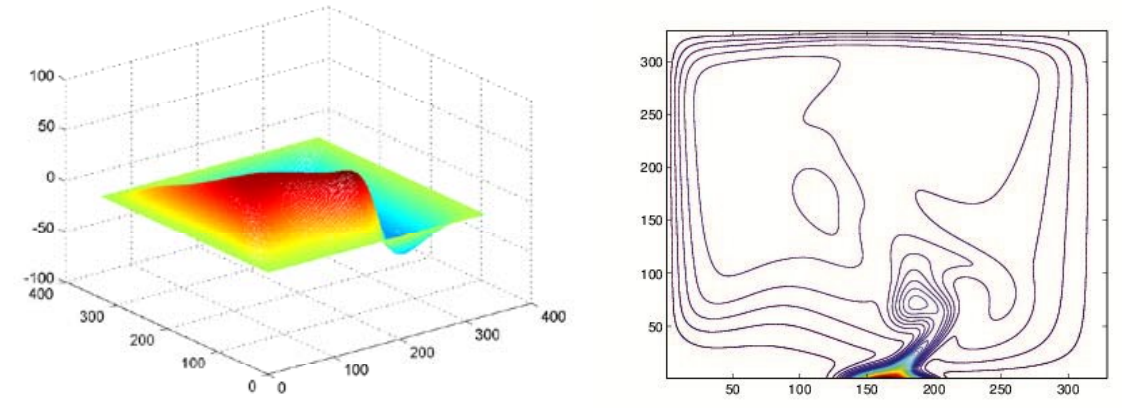

Figure 4. Stream function and isotherms for $R a=10^{10}, t=0.05, d t=0.00001, h_{x}=h_{y}=1 / 256$.

In Figure 5, we show results for $R a=10^{11}, t=0.05, \Delta t=0.00000001, h_{x}=h_{y}=1 / 328$. An additional vortex is generated, with opposite direction from the dominant vortex. This third vortex of turbulent flow, appears within the existing vortexes, and may indicate a new symmetry breaking in the flow pattern with its corresponding phase transition [5]. It should be noted that the small lateral vortex remains almost without changes during all the interval of variation of the Rayleigh number, both, in location and intensity or amplitude. The isotherms present a single pattern of full cavity with the usual characteristics presented for Rayleigh numbers less than $10^{9}$. The local loops, close to the heat source disappear, since the temperature contours, at the end of the plume, are disseminated to occupy the entire cavity with tendency to bend towards the left wall.
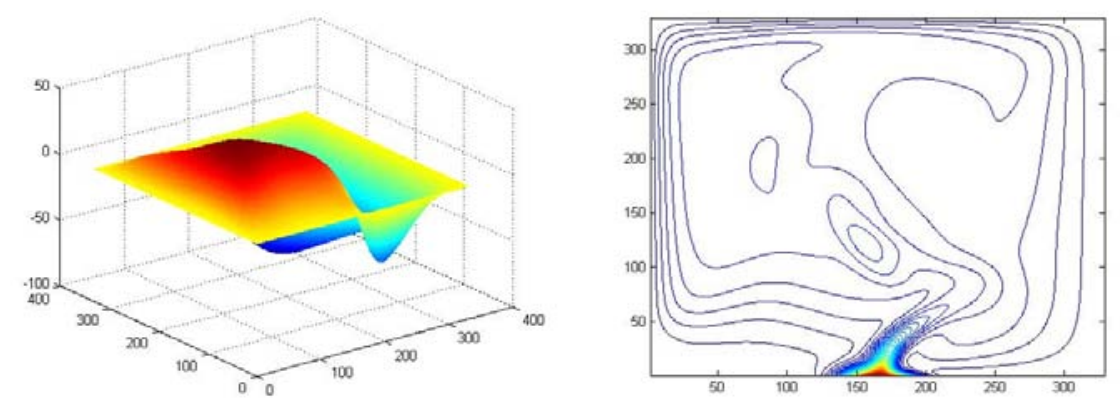

Figure 5. Stream function and isotherms for $R a=10^{11}, t=0.05, d t=0.00000001, h_{x}=h_{y}=1 / 328$. 


\section{Discussion}

We must emphasize, that although the numerical method is robust enough to handle the range of Rayleigh numbers we are dealing with, as we increase the Rayleigh number, as can be observed, a finer mesh and a smaller time step have to be used in order to obtain convergence. This implies that our computer program takes much more computing time and memory space to arrive to the final time since much more iterations have to be done and systems of linear equations of very high order have to be solved at each iteration. We are running our program in a supercomputer from the National Laboratory of Supercomputing from of the southeast of Mexico BUAP-INAOE-UDLAP (Laboratorio Nacional de Supercómputo del Sureste de México). For $R a=10^{6}$ the required time is two hours, but when working with $R a=10^{11}$, $5,000,000$ of iterations in time have to be done, and systems of linear equations of almost 108,000 variables and the same number of equations have to be solved at each iteration, so the computing time is almost four days.

\section{Conclusion}

In this paper, different turbulent flow patterns and temperature profiles, within a rectangular cavity, and for a wide range of the Rayleigh number $\left(10^{6}\right.$ to $\left.10^{11}\right)$ have been obtained. This interval for the Rayleigh number is somewhat larger, as far as we know, than those previously reported in the literature. The numerical method let us predict several phase changes in thermal convective flows. It can be observed that the isotherms describe a flow having a large asymmetric circulating region with thermal plumes induced by a local heat source at the bottom of the square cavity. These plumes are displaying an attractive influence from the right wall. Transition from quasi-steady laminar thermal behavior to structured vorticity large has been observed from the simulation. In fact, fluid flow profiles have two oppositely streamlines vortices, one almost completely dominating the flow transition in the cavity.

In summary, for $R a=10^{6}$, there exists a bifurcation in the stationary flow with two vortices in counter flow, but when increasing the Rayleigh number to $10^{7}$, the symmetry of the temperature contours is broken, and they bend towards the right wall, generating only one central vortex of large amplitude, which dominates the convective transport within the cavity. Using other numerical methods for the simulation [5], a punctual attractor in the phase space has been found in this regime of Rayleigh numbers [5].

With Ra in the range of $10^{8} \leq R a \leq 10^{9}$, a small counter flow vortex reappears, opposed to the central dominant vortex, in a specific region of the cavity. This small vortex is maintained with little variation in its amplitude during later changes of the Rayleigh number from $R a=10^{10}$ till $R a=10^{11}$. However, for $R a=10^{10}$ it distorts significantly, losing its characteristic symmetry until in $R a=10^{11}$ its amplitude decreases originating a third vortex in counter flow. This latter novel result gives us evidence that there is an additional phase transition of turbulent flow in the cavity.

All these changes in the convective flow vortices are accompanied by different breaks of symmetry in the central plume. Indeed, whenever the temperature contours are oriented towards the right wall, changes occur in the corresponding convective vortices.

Speaking about the numerical method, the fixed-point iterative process used here has shown to be robust enough to handle very high values of the parameters appearing in the Boussinesq system, such as the Rayleigh number. We are looking to improve the method with respect to the time needed for the simulation, since till now, the time needed to solve the numerical systems of equations appearing after the discretization process is very large, especially when working with high Rayleigh numbers and having to arrive to a large value of $t$ (time). At each iteration in time, this large system of linear equations is solved.

\section{Acknowledgements}

The authors would like to acknowledge the support received by the National Laboratory of Supercomputing from of the southeast of Mexico BUAP-INAOE-UDLAP (Laboratorio Nacional de Supercómputo del Sureste de México) in running our programs and especially to Dr. Enrique Varela and Dr. Manuel Martín for their support.

\section{References}

[1] Nicolás, A. and Bermúdez, B. (2005). 2D thermal/isothermal incompressible viscous flows, Int. J. Numer. Meth. Fluids, 48, pp. 349-366.

[2] Báez, E. and Nicolás, A. (2006). 2D natural convection flows in tilted cavities: porous media and homogeneous fluids. Int. J. of heat and Mass Transfer, vol. 49, 4773-4785.

[3] Desrayaud, G. and Lauriat, G. (1993). Unsteady confined buoyant plumes. J. Fluid Mechanics, vol. 252, 617-646.

[4] Minev, P. D., van de Vosse, F. N., Timmermans L. J. P., Rindt, C. C. M., van Steenhoven, A. A. (1994). Numerical 
simulation of buoyant plumes using a spectral element technique. Proceedings of Heat Transfer 94, Advanced Computational Methods in Heat Transfer, (ed. Wrobel, Brebbia and Nowak), Computational Mechanics publications, Southampton, Boston, 147-154.

[5] Basiaans, R. G. M., Rindt, C. C. M., van Steenhoven, A. A. and Nieuwstadt, F. T. M. (1998). Direct and large eddy simulation of the transition of two and three-dimensional plane plumes in a confined enclosure. Int. Journal of Heat and Mass Transfer, 41, 3989-4007.

[6] R. G. Bill Jr. and B. Gebhart. (1975). The transition of plane plumes. Int. J. Heat Mass Transfer, 18, 513-526.

[7] H. Rouse, C. S. Yih, H.W. (1952). Humphreys, Gravitational convection from a boundary source, Tellus 4, 201-210.

[8] R. Peyret and T. D. Taylor. (1983). Computational Methods for Fluid Flow, Springer-Verlag, NY.

[9] Nicolás, A. (1991). A finite element approach to the Kuramoto-Sivashinski equation, Advances in Numerical Methods, SIAM.

[10] Manar, S. M., Aljethelah, Ahmed, Al-Sammaraie, Syeda H. Tasnim, Shohel Mahmud, and Anmesh Dutta. (2018). Effect of convection heat transfer on thermal energy storage unit, Open Phys, 16: 861-867.

[11] Ahmed, S. E., Rahman, S. M., San, O., Rasheed, A., Navon, I. M. (n.d.). Memory embedded non-intrusive reduced order modelling of non-ergodic flows. Physics of Fluids, 31(12), art. no. 126602, 201.

[12] Prasopchingchana, U. (2019). Numerical study of natural convection in a rectangular cavity with variation of cavity aspect ratios and cavity inclination angles, IOP Conference Series: Materials Science and Engineering, 576(1), art. no. 012044.

[13] Mamourian, M., Shirvan, K. M., Ellahi, R., Rahimi, A. B. (2016). Optimization of mixed convection heat transfer with entropy generation in a wavy surface square lid-driven cavity by means of Taguchi approach. Int. J. Heat Mass Transf., 102, 544-554.

[14] Mastiani, M., Kim, M. M., Nematollahi, A. (2017). Density maximum effects on mixed convection in a square lid-driven enclosure filled with Cu-water nanofluids. Adv. Powder Technol., 28, 197-214.

[15] Yu, Q., Xu, H., Liao, S. J. (2018). Analysis of mixed convection flow in an inclined lid-driven enclosure with Buongiorno’s nanofluid model. Int. J. Heat Mass Transf., 126, 221-236.

[16] Lei, Wang, Wei-Wei Wang, Yang Cai, Fu-Yun Zhao. (2020). Effects of porous fins on mixed convection and heat transfer mechanics in Lid-Driven cavities: Full modelling and parametric simulations. Transport in Porous Media, 132:495:534. 